\title{
Compact Nuclei of Galaxies and Sources of Their Energy
}

\author{
L.Sh. Grigoryan \\ G.S. Sahakian \\ Yerevan State University, Yerevan, 375049, Armenia
}

Institute of Applied Problems in Physics, Yerevan, 375014, Armenia

A model of compact nuclei of galaxies as spherically-symmetric star clusters is proposed. A concept of the equation of state for star clusters in statistical equilibrium is introduced (galactic nuclei are systems in statistical equilibrium if their age is of the order of the age of the Universe). It is shown that a statistically equilibrium star cluster is described by the equation of state of a polytrope $P=a \rho^{3}$, and with its help the main parameters of compact nuclei of galaxies are calculated. The formula $M=2.524 G R^{5} / a$ for mass $M$ and radius $R$ of the cluster is derived.

From the law of equipartition of internal energy among the degrees of freedom of stars (including the rotational degrees of freedom), it is proved that the main constituents of compact galactic nuclei are neutron stars and white dwarfs. The ordinary stars will be destroyed because of relatively fast rotation. The angular velocities of neutron stars in the nucleus of our Galaxy corresponding to the condition of statistical equilibrium are approximately the same as those observed for pulsars. Hence the nucleus of the Galaxy may be a possible source of pulsars. It is shown that $1.5 \%$ of stars can evaporate from the nucleus of our Galaxy. The number of pulsars among such stars is of the order of $3 \cdot 10^{6}$. The problem of energy sources of galactic nuclei is also discussed. It is shown that in the framework of the model under consideration the compact nuclei of galaxies are high power sources of hard $\gamma$-radiation $\left(L \approx 10^{48} N_{8} \mu_{30}^{2}(\Omega / 50)^{3} \mathrm{erg} / \mathrm{s}\right.$, where $\mu$ is the magnetic moment, $\Omega$ - the angular velocity of neutron star rotation and $N$ the number of stars in the galactic nucleus) due to the curvature radiation from ultrarelativistic electron fluxes traversing along the channels of open magnetic lines of pulsars. The X-ray and ultraviolet radiation of galactic nuclei are due to the synchrotron radiation from these same electron fluxes in the magnetic field of galactic nuclei $\left(L \approx 10^{42}-10^{44} \mathrm{erg} / \mathrm{s}\right)$. The optical (ranges of visible and infrared radiation) and the radio-frequency radiation are caused by the bremsstrahlung from the electrons of interstellar space $\left(L \approx 6 \cdot 10^{46} N_{8}^{2}\left(5 / R_{p c}\right)^{3} \mathrm{erg} / \mathrm{s}\right.$, where $R$ is the radius of the galactic nucleus). 